\title{
CARDIOVASCULAR RESPONSES TO CEREBRAL ISCHEMIA FOLLOWING BILATERAL CAROTID ARTERY OCCLUSION IN SHRSP, SHRSR AND WKY RATS
}

\author{
Mitsuru KAKIHANA, Akio SHINO and Akinobu NAGAOKA \\ Biology Laboratories. Central Research Division, Takeda Chemical Industries LId., \\ 17-85. Jusohonmachi 2-chome, Yodogawa-ku. Osaka 532. Japan
}

Accepted July 31,1982

\begin{abstract}
Systemic arterial pressure was markedly increased in the early phase of cerebral ischemia induced by bilateral carotid artery occlusion (BCAO) in stroke-prone spontaneously hypertensive rats (SHRSP). The elevated level of arterial pressure was gradually returned to the initial level, and hypotension followed in the late phase. Severe neurological symptoms such as "ischemic seizure", dyspnea and coma were developed in the late phase. All SHRSP died within $6 \mathrm{hr}$ after BCAO. The heart rate continued to increase during the brain ischemia. Cardiac arrhythmias, significant increases in plasma levels of creatine phosphokinase (CPK) and CPK-MB isozyme and disruption of myofibrils were observed after BCAO, particularly after the development of ischemic seizure. In contrast, in stroke-resistant SHR (SHRSR) and Wistar-Kyoto rats (WKY), ischemic seizure did not develop, yet all died within $8 \mathrm{hr}$ after BCAO. Arterial pressures were moderately increased and never decreased to below the initial levels during the observation periods. Increases in CPK-MB isozyme activities in plasma from SHRSR and WKY were not detected. Pretreatments with propranolol and reserpine inhibited the increases in heart rate, reduced the frequency of arrhythmias and prolonged the survival time following BCAO in SHRSP. Our results indicate that cardiac dysfunction, which is a consequence of the cerebral ischemia, may be one of the causes of death following $B C A O$ in SHRSP.
\end{abstract}

Bilateral carotid artery occlusion (BCAO) causes a marked brain ischemia in spontaneously hypertensive rats (SHR) and a stroke-prone strain of SHR (SHRSP) (1-4). In both these strains of SHR, after BCAO. cerebral anaerobic metabolites were increased and adenosine triphosphate were decreased leading to the development of various neurological symptoms. Clinical studies (5-11) in patients with acute cerebrovascular accidents demonstrated a high incidence of cardiac arrhythmias and other electrocardiographic (ECG) abnormalities, histological changes in the myocardium, and elevated levels of serum cardiac enzymes such as creatine phosphokinase (CPK) and lactate dehydrogenase
( $\mathrm{LDH}$ ). Dimant and Grob (10) reported that twenty-nine percent of the patients with stroke had raised serum enzyme levels, and those with elevated CPK levels had a higher incidence of ECG abnormality as well as a higher mortality than those with normal CPK levels.

In the present study, we attempted to elucidate the relationship between the alteration of the cardiovascular system and the development of neurological deficits following BCAO in the SHRs and control WistarKyoto rats (WKY). We examined the changes in systemic arterial pressure, heart rate, ECG, plasma levels of cardiac enzymes and isazyme patterns, histological changes in the myo- 
cardium, and ischemic neurological deficits in conscious rats. Effects of antiadrenergic drugs on these so-induced changes were evaluated.

\section{Materials and Methods}

General: Male stroke-prone spontaneously hypertensive rats (SHRSP), a stroke-resistant SHR (SHRSR) and nor motensive Wistar-Kyoto rats (WKY), 10-11 weeks of age, were used. The rats had been raised in our laboratories and given a laboratory chow. CE-2, Japan Clea and tap water ad libitum. Under light ether anesthesia. the bilateral common carotid arteries were exposed through a ventral midline incision in the neck and were separated carefully from vagosympathetic trunks. When the rats had recovered from ether anesthesia after $5 \mathrm{~min}$. the arteries were simultaneously ligated with silk sutures, and the skin incision was closed with steel clips. Neurological symptoms after BCAO were scored as follows: 0 , normal: 1, slight decrease in spontaneous motor activity: 2 , no ambulation and weakness of hindlimb: 3, weakness of all four limbs, and presence of righting reflex: 4 . weakness of all limbs, and no lighting reflex; 5, piloerection and tremor; 6, jumping and/or seizure (ischemic seizure): 7 , dyspnea or coma; 8, death.

Hemodynamics and neurological changes: Systemic arterial pressure, heart rate and ischemic neurological symptoms following BCAO were examined in SHRSP $(N=8)$. SHRSP $(N=6)$ and WKY $(N=6)$. The femoral artery was cannulated with a polyethylene tube (PE-10) filled with sodium heparin $(100 \mathrm{lU} / \mathrm{ml}$ in $0.9 \% \mathrm{NaCl})$. This tube was reflected dorsally and anteriorly. led subcutaneously to the back of the neck, and exteriorized through a small skin puncture. The catheter was flushed occasionally with saline containing $100 \mathrm{IU}$ sodium heparin per $\mathrm{ml}$ to prevent blood clotting. The rats were placed individually in a transparent plastic cylinder (22 cm diam. $55 \mathrm{~cm}$ height). Mean arterial pressure was measured via a femoral arterial catheter with a pressure transducer (MPU-0.5) and polygraph (RM-45. Nihon Koden, Tokyo). Heart rate was calculated from the blood pressure tracing. Thirty minutes after surgery, mean arterial pressure and heart rate were recorded as the initial values. The changes in blood pressure and heart rate and the development of neurological symptoms were observed for $8 \mathrm{hr}$ and at $24 \mathrm{hr}$ after BCAO. Because jumping and/or seizure was the most unequivocal symptom (termed ischemic seizure), the time which elapsed until onset of the ischemic seizure and death were recorded. In rats with no ischemic seizure up to $8 \mathrm{hr}$, the time was recorded as $480 \mathrm{~min}$.

Plasma enzymes: Changes in plasma lactate dehydrogenase (LDH) and creatine phosphokinase (CPK) contents and thier isozyme patterns after BCAO were determined in SHRSP $(N=20)$. SHRSR $(N=15)$ and WKY $(N=15)$. Sham-operated rats were used as controls. Under ether anesthesia, the blood of each rat was sampled from the abdominal aorta. The plasma was stored at $-20^{\circ} \mathrm{C}$ until assayed. CPK and LDH contents were estimated with the activated ultraviolet test set of Boehringer (Mannheim) and the LDH test set of Wako (Osaka), respectively. CPK and LDH isozymes were fractionated by agarose electrophoresis (CPK isozymes, Sigma 715-PE. St. Louis) and agar electrophoresis (LDH isozyme-test, Wako, Osaka), respectively. Both CPK and LDH isozymes were visualized by formation of formazan. Quantitation of CPK-isozymes was accomplished by colorimetric scanning using a densitometer (TLC scanner, Shimadzu CS. 910, Kyoto)

Electrocardiogram: Changes in electrocardiogram (ECG) following $\mathrm{BCAO}$ were recorded in five conscious SHRSP since only 
SHRSP showed a marked change in arterial pressure in the preliminary experiments. Limb leads were constructed from small needle electrodes placed subcutaneously at the fore-limbs and the left hind-limb, and the electrodes were fixed with adhesivetape. The femoral artery was cannulated as described above. Systemic arterial pressure and ECG (lead II) were recorded simultaneously on a polygraph.

Effects of antiadrenergic drugs: Effects of antiadrenergic drugs on the development of cardiovascular and neurological abnormalities were observed in SHRSP. Twenty-four rats were assigned to four groups. DL-propranolol hydrochloride at $2 \mathrm{mg} / \mathrm{kg}$. phentolamine mesylate at $5 \mathrm{mg} / \mathrm{kg}$ or saline at $2.5 \mathrm{ml} / \mathrm{kg}$ was subcutaneously administered $30 \mathrm{~min}$ before BCAO. Reserpine at $2 \mathrm{mg} / \mathrm{kg}$ and 1 $\mathrm{mg} / \mathrm{kg}$ were subcutaneously administered about $16 \mathrm{hr}$ and $30 \mathrm{~min}$ before BCAO. respectively. Mean arterial pressure and heart rate were recorded via a femoral arterial catheter implanted $1 \mathrm{hr}$ before BCAO. The transient, precipitous fall in blood pressure was regarded as a sign of ventricular arrhythmias occurring between BCAO and the development of ischemic seizure, and the severity of arrhythmias was represented as a frequency per $10 \mathrm{~min}$. Changes in neurological symptoms and ECG were observed for $8 \mathrm{hr}$ after BCAO. In rats with no ischemic seizure up to $8 \mathrm{hr}$, the onset-time of ischemic seizure was recorded as $480 \mathrm{~min}$.

Histological examination: Histological ex- amination was carried out with the hearts from SHRSP which were sacrificed at 0,30 , 90 and 180 min after BCAO. The hearts were fixed with buffered $10 \%$ formalin and embedded in paraffin. Sections of $5 \mu$ thickness were cut and stained with hematoxylin and eosin.

Statistical analysis: Statistical differences were evaluated using the Mann-Whitney $U$ test for plasma enzyme levels and for the time required for the onset of ischemic seizure and death following BCAO, and the Student's $t$-test was used for the others. All values were expressed as the mean \pm S.E.M.

\section{Results}

Hemodynamics and neurological changes: Mean arterial pressures before BCAO were $166 \pm 5,126 \pm 4$ and $107 \pm 2 \mathrm{mmHg}$ in SHRSP. SHRSR and WKY, respectively (Table 1). Heart rates were similar in the three strains. In SHRSP, there were observed a decrease in spontaneous motor activity and a marked weakness of all the limbs (score 1-3) within $60 \mathrm{~min}$ after $\mathrm{BCAO}$. The time required for onset of ischemic seizure (score 6) was $112 \pm 10 \mathrm{~min}$ (Table 1). Thereafter, the animals became dyspneic or comatose (score 7) and died within $6 \mathrm{hr}$ after BCAO, with an average death time of $248 \pm 16 \mathrm{~min}$. In contrast, in SHRSR and WKY, ischemic seizure did not develop, yet all died within 8 hr after BCAO. Some of SHRSR and WKY showed only slight neurological abnormalities (score 1-3).

Table 1. Initial mean arterial pressure (MAP), heart rate (HR) and time required for onset of ischemic seizure and survival time followirg bilateral carotid artery occlusion (BCAO) in WKY. SHRSR and SHRSP

\begin{tabular}{lccc}
\hline & WKY & SHRSR & SHRSP \\
MAP (mmHg) & $107=2$ & $126 \pm 4^{*}$ & $166 \pm 5^{*} \pm$ \\
HR (beats/min) & $384 \pm 18$ & $361 \pm 13$ & $367 \pm 10$ \\
Onset time of ischemic scizure $(\mathrm{min})$ & $>480$ & $>480$ & $112 \pm 10^{*}=$ \\
Survival time (min) & $>480$ & $>480$ & $248 \pm 16^{*}=$ \\
Number of rats & 6 & 6 & 8 \\
\hline
\end{tabular}

${ }^{*} \mathrm{P}<0.001$ vs WKY, $\$ P<0.001$ vs SHRSR. Values are the mean \pm S.E.M. 


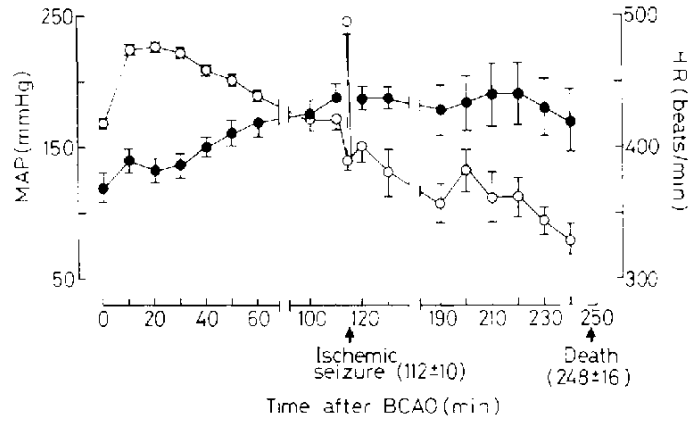

Fig. 1. Changes in mean arterial pressure (MAP) and hoart rate (HR) following BCAO in SHRSP. MAP, O-O; HR, - Each point is the mean= S.E.M. of 8 rats. Time-course of MAP and HR changes are constructed from three periods: left, the first 1 -hr period following BCAO; center, the 30-min period before and after the first development of ischemic seizure: right, the 1 -hr period before death following BCAO. MAR and HR were determined every 10 min in each period.

Time-course of arterial pressure changes following BCAO in SHRSP is shown in Fig. 1. and a representative recording is given in Fig. 2. In the early phase of cerebral ischemia, mean arterial pressure rose markedly immediately after BCAO, and reached a level of $222 \pm 3 \mathrm{mmHg} 10 \mathrm{~min}$ later. The elevated level lasted for about $30 \mathrm{~min}$ and then returned to the initial level within $90 \mathrm{~min}$. in the late phase of cerebral ischemia, transient but marked elevation of blood pressure was observed at the time when the ischemic seizure developed. The average increment was about $100 \mathrm{mmHg}$ at the first development of the seizure (Fig. 1). After the repeated occurrence of ischemic seizure, arterial pressure was further decreased to hypotensive levels. Heart rate continued to increase during the brain ischemia (Fig. 1). Arterial pressure and heart rate in SHRSR and WKY were moderately increased and never decreased to below the initial levels. In SHRSR, mean arterial pressure gradually increased to $175-180 \mathrm{mmHg}$ at $40-80 \mathrm{~min}$ after $\mathrm{BCAO}$ and returned to the initial level within $8 \mathrm{hr}$. In WKY, the arterial pressure

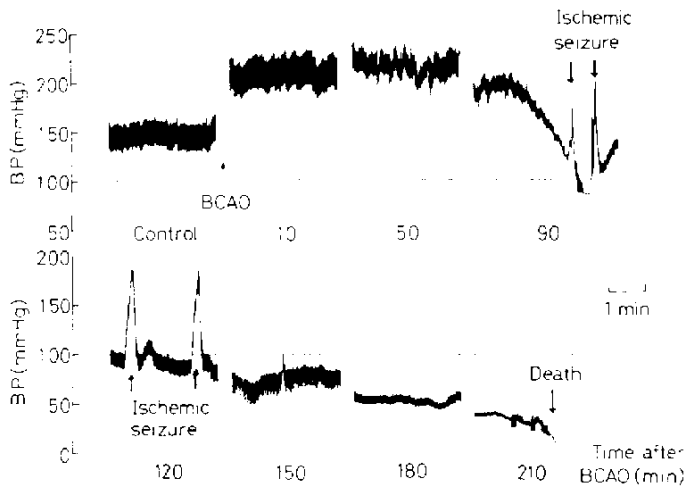

Fig. 2. Representative change in blood pressure following BCAO in SHRSP.

slightly increased to a level of about 130 $\mathrm{mmHg}$ at $20 \mathrm{~min}$ after BCAO. The elevated level continued during the observation period. Heart rate in these strains of rats slightly increased, but returned to the initial level within $3 \mathrm{hr}$.

Plasma enzyme activities: Alterations in plasma LDH and CPK activities and LDH and CPK isozyme patterns after BCAO in SHRSP. SHRSR and WKY are shown in Table 2 and Figs. 3 and 4. In SHRSP, both total LDH and CPK levels were significantly increased after $\mathrm{BCAO}$. Increases in the levels were much more pronounced at 3 than $1 \mathrm{hr}$ after $\mathrm{BCAO}$. $\mathrm{LDH}_{1}$ and $\mathrm{LDH}_{2}$ isozymes not detectable in the plasma of sham-operated controls, were identified (Fig. 3). Moreover, a marked increase in CPK-MB isozyme was observed in SHRSP, particularly in the SHRSP with severe neurological deficits (score 7) and hypotension (Figs. 4 and 5).

Total LDH and CPK levels in WKY and SHRSR were increased slightly but significantly. Increases in cardiac enzymes such as $\mathrm{LDH}_{1}$ and $\mathrm{LDH}_{2}$ isozymes or CPK-MB isozyme in plasma from SHRSR and WKY were nil (Figs. 3 and 4).

Electrocardiogram: A transient but marked fall in blood pressure (Fig. 2, at 50 min of $\mathrm{BCAO}$ ) sometimes followed BCAO in the SHRSP. When the transient and precipitous 
Table 2. Changes in plasma lactate dehydrogenase (LDH) and creatine phosphokinase (CPK) activities following BCAO in WKY, SHRSR and SHRSP

\begin{tabular}{|c|c|c|c|c|}
\hline Enzyme & $\begin{array}{l}\text { Time (hr) } \\
\text { after BCAO }\end{array}$ & WKY & SHRSR & SHRSP \\
\hline & Control & $180 \pm 28 \quad(N=5)$ & $161 \pm 11(N=5)$ & $165 \pm 10(N=5)$ \\
\hline $\mathrm{LDH}$ & 1 & $313 \pm 31(N=5)^{*}$ & $176 \pm 9(N=5)$ & $296 \pm 45(N=5)^{*}$ \\
\hline \multirow[t]{2}{*}{$(\mathrm{U} / \mathrm{ml})$} & 3 & $162 \pm 15(N=4)$ & $189 \pm 16(N=5)$ & $688 \pm 86(N=9)^{* * *}$ \\
\hline & Control & $31=4(N=5)$ & $32 \pm 1(N=5)$ & $34 \pm 3(N=5)$ \\
\hline CPK & 1 & $40 \pm 8(N=5)^{*}$ & $41 \pm 3(N=5)^{*}$ & $57 \pm 11(N=5)^{* *}$ \\
\hline$(U / 1)$ & 3 & $59 \pm 4(N=4)^{*}$ & $37 \pm 1(N=5)^{*}$ & $264+50(N-9)^{* * *}$ \\
\hline
\end{tabular}

${ }^{*} \mathrm{P}<0.05,{ }^{* *} \mathrm{P}<0.01,{ }^{* *} \mathrm{P}<0.001$ vs the control. ( ) =number of rats, Values aro tho mean $\pm S . \mathrm{E} . \mathrm{M}$.

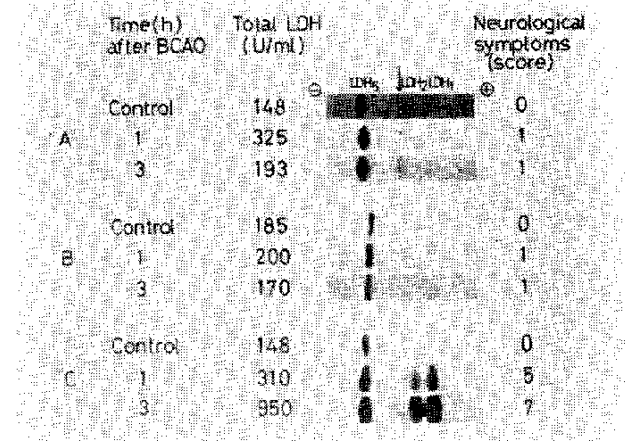

Fig. 3. Plasma lactato dehydrogenase isozyme patterns 1 and 3 hr after BCAO in WKY (A), SHRSR (B) and SHRSP (C). The origin is indicated by the arrow. Neurclogical symptoms: scorc 0 , normal: score 1. slight decrease in spontancous motor activity: score 5, piloerection and tremor; score 7. dyspnea or coma.

fall of blood pressure (Fig. 6, left) and seizures (Fig. 6, right) occurred, cardiac rhythm disturbances such as single and mutiple ventricular premature beats were observed. These arrhythmias were noted in every SHRSP so-tested.

In an attempt at elucidating the effect of antiadrenergic drugs on the occurrence of cardiac arrhythmias in SHRSP, the frequency of the transient and precipitous fall of blood pressure between BCAO and development of ischemic seizure was determined to assess the severity of the cardiac arrhythmias.

Effects of antiadrenergic drugs: Effects of antiadrenergic drugs on mean arterial pressure, heart rate, frequency of arrhythmias and

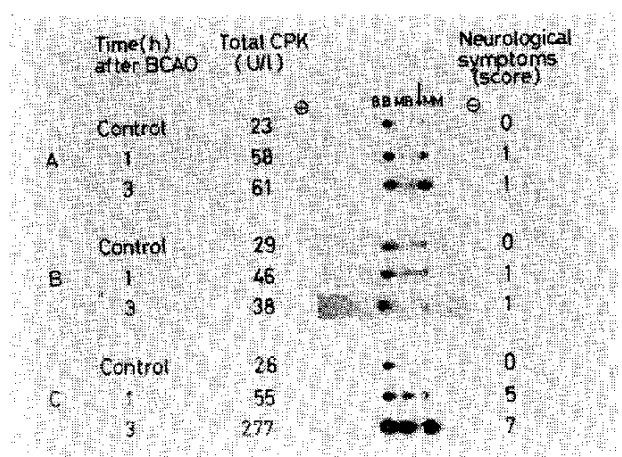

Fig. 4. Plasma creatine phosphokinase isorymo patterns 1 and 3 hr after BCAO in WKY (A). SHRSR (B) and SHRSP (C). The origin is indicated by the arrow. Neurological symptoms: score 0 , normal: score 1. slight decrease in spontaneous motor aclivity: score 5, piloerection and tremar; score 7. dyspnea or coma.

onset-times of ischemic seizure and death following BCAO in SHRSP are summarized in Table 3. Propranolol and reserpine decreased the heart rate, reduced the frequency of arrhythmias and prolonged the survival time. In addition, reserpine decreased the pressor response and prolonged the onsettime of ischemic seizure. On the other hand, phentolamine inhibited the pressor response. but not the frequency of cardiac arrythmias: As a result, the onset-time of ischemic seizure was accelerated, and the elapse of time to death was tended to be shortened.

Histological findings in the heart: The hearts of SHRSP showed spontaneous, slight lesions such as cytoplasmic micro- 
vacuolation, focal or single cell necrosis and focal fibrosis (Table 4). At $30 \mathrm{~min}$ after BCAO, a slight increase in cytoplasmic vacuoles was observed in one out of three SHRSP. In the late phase of BCAO, degeneration of cardiocytes which showed focal disruption or loss of myofibrils was detected.

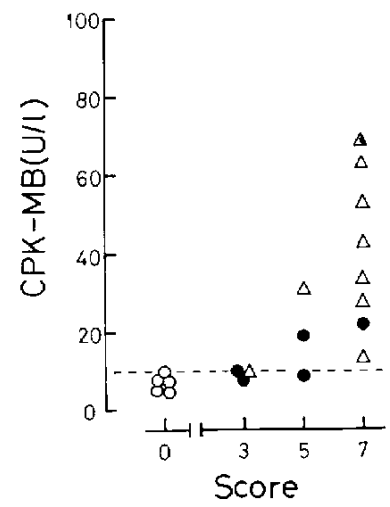

Fig. 5. Relationship between neurological symptoms and plasma CPK-MB isozyme activitiy following BCAO in SHRSP. Neurological symptoms: score 0 . normal: score 3. weakness of all limbs: score 5. piloerection and tremor; score 7. dyspnea or coma. The difference of CPK-MB activity between score 0 and score 7 is statistically significant $(P<0.001)$. O. control: $1 \mathrm{hr}$ after $\mathrm{BCAO}$ : $\triangle 3$ hr after BCAO.
The myofibrilar degeneration was more prominent in the SHRSP sacrificed at 180 min (Fig. 7, Table 4).

\section{Discussion}

The pathogenetic mechanism of cerebral ischemia after BCAO in SHR has been studied by Fujishima et al. (1-3, 12, 13). They found that the increased vascular resistance due to persistent high blood pressure in SHR might be responsible for an upward shift of cerebral blood flow auto-

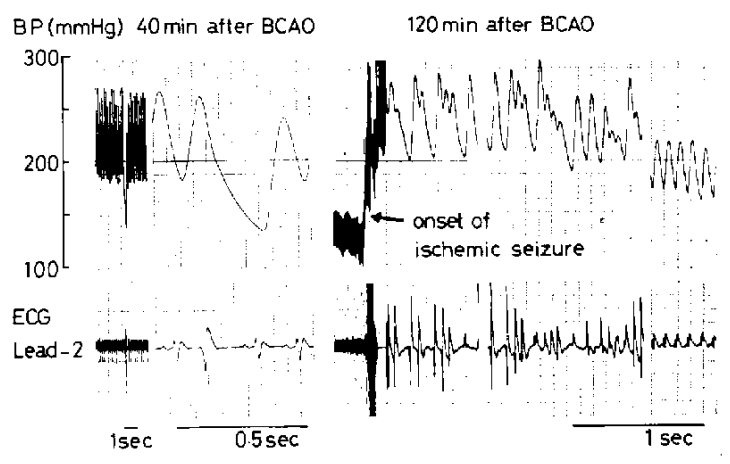

Fig. 6. Representative recording of cardiac arrhythmias following BCAO in SHRSP.
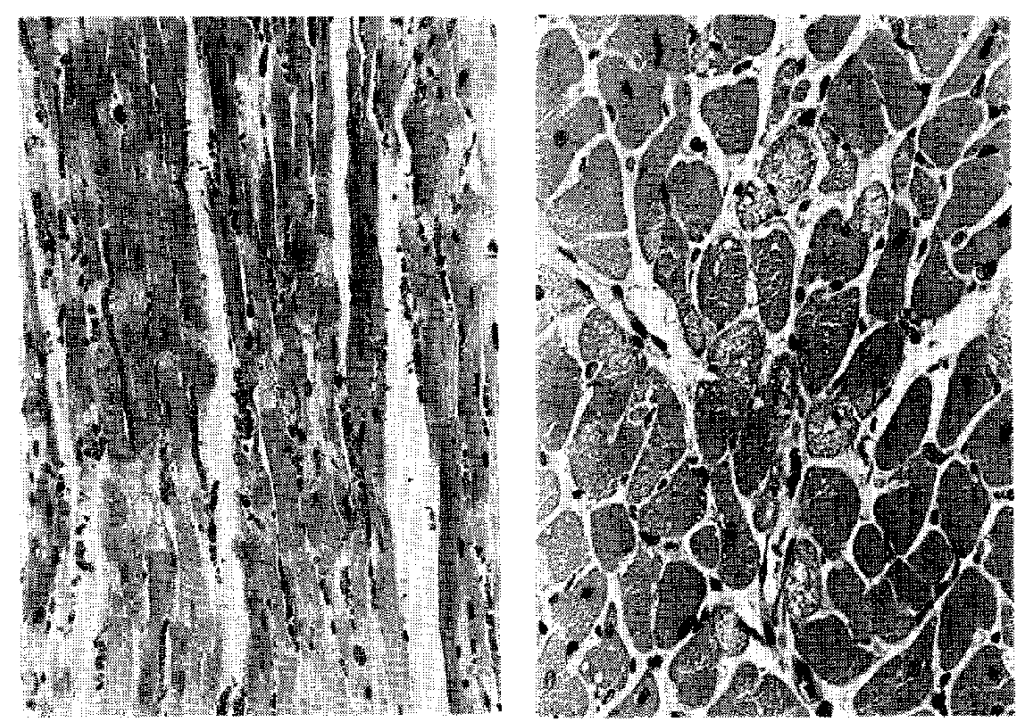

Fig. 7. Cardiocyte of SHRSP sacrificed 180 min after BCAO. Many cardiocytes show disruption or loss of myofibrils in both transcverso and longitudinal sections. H.E. stain. Left: transeverso section. $\times 260$. Right: longitudinal section, $\times 520$. 

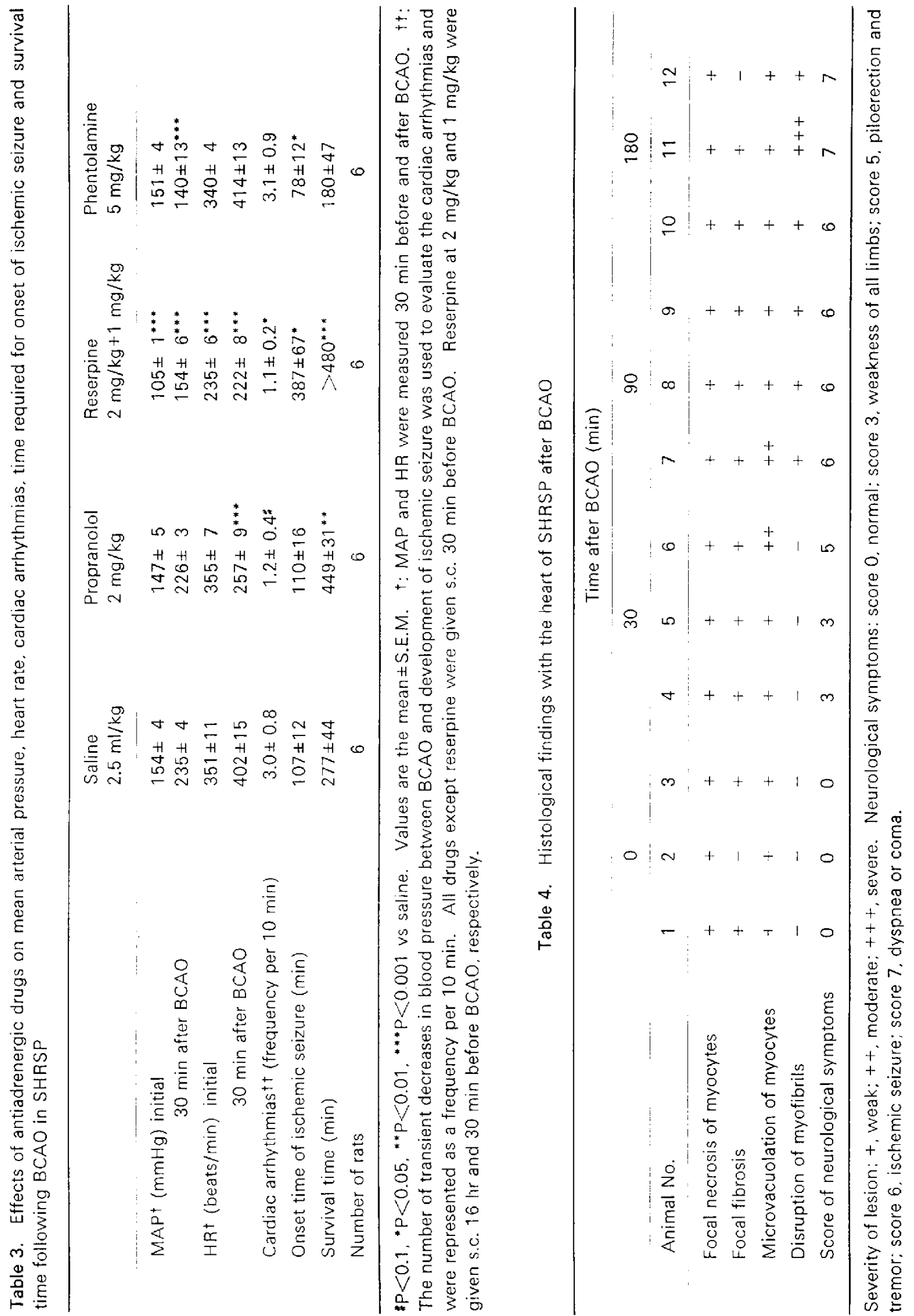
regulation and a marked reduction of the cerebral perfusion pressure after BCAO, resulting in severe brain ischemia. The stroke-prone strain of SHR (SHRSP) shows a steeper rise of blood pressure at an early stage of hypertension (14). It has been also found that the distribution of cerebral arteries is essentially the same between SHR and WKY (15) and between SHRSP and WKY (16). These findings indicate that a functional mechanism in cerebral circulation rather than a morphological one may account for the increased sensitivity to brain ischemia in the SHRs. In a previous study (17), in which neurological symptoms and cerebral metabolic changes after BCAO were observed in SHRSP, SHRSR, WKY and thier $F_{1}$ and $F_{2}$ hybrids, we showed that not only hypertension but also genetic factors may play an important role in the susceptibility to brain ischemia in SHRSP.

It is well known that the primary and acute response to brain ischemia consists of arterial hypertension, bradycardia and apnea $(18-22)$. The rise in arterial pressure is due to an increased peripheral vasoconstriction which is primarily neurogenic and mediated by $\alpha$-adrenergic receptors. Recently, Dampney et al. (23) reported that the pressor response to cerebral ischemia, caused by clamping both common carotid arteries after previously occluding the vertebral arteries of anesthetized rabbits, was mediated by a restricted region in the medulla oblongata. In the present experiment, characteristic responses in the cardiovascular system to BCAO were evident in stroke-prone SHR. Systemic arterial pressure was markedly elevated in the early phase of brain ischemia. The pressor response was inhibited by an $\alpha$ blocker, phentolamine, but not a $\beta$-blocker, propranolol. Bradycardia and apnea, however. could not be observed in the early phase of brain ischemia in SHRSP. This difference in cardiovascular response to cerebral ischemia seems to be due to the difference in the type of cerebral ischemia. In the previous studies, most experiments were performed under the condition of almost complete ischemia. In SHRSP with BCAO, the regional blood flow in the cerebral cortex was decreased to about $20 \%$ of the basal value and that in the brainstem including the medulla oblongata did not change in the early phase of cerebral ischemia (4). Further work will be needed to elucidate the detailed neural mechanism involved in the cardiovascular responses to cerebral ischemia following BCAO in SHRSP.

Knowledge of the level of serum cardiac enzymes ( $L D H . C P K$ etc.) is pertinent to evaluate the extent of cardiac damage. Of these enzymes, the serum levels of CPK and CPK-MB isozyme proved to be the most usefull indicators of myocardial damage (24. 25). A growing body of clinical and experimental data revealed that cerebrovascular lesions exhibit a high incidence of ECG abnormalities and arrhythmias with elevated serum levels of cardiac enzymes (11, 12, 26. 27). Plasma levels of $L D H$ and CPK were markedly increased after BCAO in SHRSP. The degree of increases in CPK-MB tended to be correlate with severity of the neurological symptoms. In addition, cardiac arrhythmias was detected during the period of brain ischemia. particularly in the late phase. These findings strongly suggest that brain ischemia induces myocardial dysfunction in SHRSP with BCAO. In fact, disruption of myofibrils was found in the late phase of cerebral ischemia in SHRSP.

The marked elevation of systemic arterial pressure in SHRSP with BCAO was followed by gradual return to the initial level in the early phase of brain ischemia. After repeated occurrence of ischemic seizure, arterial pressure was further decreased to a hypotensive level in the late phase. It should be stressed that cardiac rhythm disturbances and release of CPK-MB isozyme from the myocardium 
were detected in the early phase of brain ischemia. Moreover, focal myocardial necrosis and fibrosis were observed in SHRSP with and without BCAO. Yamori et al. (28) have already reported that genetic hypertension accompanied by left ventricular hypertrophy with thickening of coronary arterial wall may be a pathogenetic factor for myocardial lesions or myocardial infarction in SHRSP. These pathophysiological changes in the heart of SHRSP may explain the increased susceptibility to myocardial damage due to cerebral ischemia, leading to the development of arrhythmias, the release of cardiac enzymes, and the breakdown of pressor response (compensatory response) in the SHRSP.

It has been reported $(28-30)$ that hearts subjected to coronary artery occlusion are hyperreactive to arrhythmogenic effects of catecholamines and that reserpine and propranolol prevent the occurrence of cardiac arrhythmias in such hearts. These drugs also limited the enzyme release from the myocardium in the experimental ischemic and hypoxic hearts $(31-34)$. In addition, in clinical and experimental studies (35-39). propranolol and reserpine ameliorated the ECG changes and myocardial lesions occurring in cerebrovascular accidents. In the present study, pretreatment with reserpine and propranolol reduced the frequency of cardiac arrhythmias and prolonged the survival time after BCAO in SHRSP. All these findings suggest that the disturbance of cardiac function may be one of the causes of death after BCAO in SHRSP.

Acknowledgment: We thank M. Ohara of Kyushu University and Dr. K. Kikuchi for comments on the manuscript.

\section{References}

1) Fujishima, M., Sugi, T., Morotomi, Y. and Omae, T.: Effects of bilateral carotid artery ligation on brain lactate and pyruvate concentrations in normotensive and spontaneously hypertensive rats. Stroke 6, 62-63 (1975)

2) Fujishima, M. and Omae, T.: Cerebral lactate, pyruvate and ATP concentrations, and arterial acid-base balance at various time intervals following bilateral carotid artery occlusion in normotensive and spontaneously hypertensive rats. Acta Neurol. Scand. 54, 13-21 (1976)

3) Choki, J., Yamaguchi, T., Takeya, Y., Morotomi, $Y$, and Omae, T.: Effect of carotid artery ligation on regional cerebral blood flow in normotensive and spontaneously hypertensive rats. Stroke 8 , 374-379 (1977)

4) Kakihana, M., Suno, M., Shibota, M., Fujiwara, K., Hamajo, K. and Nagaoka, A.: Changes of behavior, cerebral blood flow and metabolism after bilateral carotid artery occlusion in strokeprone SHR, stroke-resistant SHR and normal Wistar-Kyoto rats. Japan. Heart J. 21, 569 (1980)

5) Lavy, S., Stern, S., Herishianu, Y, and Carmon, A.: Electrocardiographic changes in ischemic stroke. J. Neurol. Sci. 7, 409-415 (1968)

6) Lavy, S., Yaar, I., Melamed, E. and Stern, S.: Effect of acute stroke on cardiac functions as observed in an intensive care unit. Stroke 5 , 775-780 (1974)

7) Norris, J.W., Froggatt, H.B. and Hachinski, V.C.: Cardiac arrhythmias in acute stroke. Stroke 9, 392-396 (1978)

8) Conner, R.C.R.: Focal myocytolysis and fuchsinophilic degeneration of the myocardium of patients dying with various brain lesions. Ann. N.Y. Acad. Sci. 156, 261-270 (1969)

9) Hammermeister, K.E. and Reichenbach, D.D.: QRS change, pulmonary edema, and myocardial necrosis associated with subarachnoid hemorrhage. Am. Heart J. 78, 94-100 (1969)

10) Dimant, J. and Grob, D.: Electrocardiographic changes and myocardial damage in patients with acute cerebrovascular accidents. Stroke 8 , 448-455 (1977)

11) Norris, J.W., Hachinski, V.C., Myers, H.G., Callow, J., Wong, T. and Moore, R.W.: Serum cardiac enzymes in stroke. Stroke 10, 548-553 (1979)

12) Fujishima, M. and Omae, T.: Lower limit of cerebral autoregulation in normotensive and spontaneously hypertensive rats. Experientia 32, 1019-1021 (1976)

13) Fujishima, M. and Omae, T.: Carotid back pressure following bilateral carotid occlusion in normotensive and spontaneously hypertensive rats. Experientia 32, 1021-1022 (1976)

14) Nagaoka, A., Iwatsuka, H., Suzuoki, Z. and Okamoto, K.: Genetic predisposition to stroke in spontaneously hypertensive rats. Am. J. 
Physiol. 230, 1354-1359 (1976)

15) Ogata, J., Fujishima, M., Morotomi, Y. and Omae, T.: Cerebral infarction following bilateral carotid artery ligation in normotensive and spontaneously hypertensive rats: a pathological study. Stroke 7, 54-60 (1976)

16) Yamori, Y., Horie, R., Handa, H., Sato, M. and Fukase, M.: Pathogenetic similarity of strokes in stroke-prone spontaneously hypertensive rats and humans. Stroke 7, 46-53 (1976)

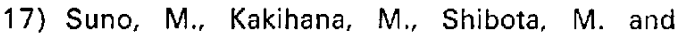
Nagaoka, A.: Mechanism of increased sensitivity to cerebral ischemia following carotid artery occlusion in stroke-prone spontaneously hypertensive rats: Importance of genetic factors. Stroke 12, 246-250 (1981)

18) Guyton, A.C.: Acute hypertension in dogs with cerebral ischemia. Am. J. Physiol. 154, 45-54 (1948)

19) Sagawa, K., Ross, J.M. and Guyton, A.C. Quantitation of cerebral ischemic pressor response in dogs. Am. J. Physiol. 200, 11641168 (1961)

20) Downing, S.E., Mitchell, J.H. and Wallace, A.G.: Cardiovascular responses to ischemia, hypoxia and hypercapnia of the central nervous system. Am. J. Physiol. 204, 881-887 (1963)

21) Miyakawa, K.: A method of complete interception of the blood supply to the brain of the rabbit. Med. J. Shinshu Univ. 11, 105-112 (1966)

22) Lavy, M.N., Ng, M.L. and Zieske, H.: Cardiac response to cephalic ischemia. Am. J. Physiol. $215,169-175$ (1968)

23) Dampney, R.A.L., Kumada, M. and Reis, D.J.: Central neural mechanism of the cerebral ischemic response. Circ. Res. 44, 48-62 (1979)

24) Sobel, B.E. and Shell, W.E.: Serum enzyme determination in the diagnosis and assessment of myocardial infarction. Circulation 45, 471482 (1972)

25) Wagner, G.S., Roe, C.R., Limbird, L.E., Rosati, R.A. and Wallace, A.G.: The importance of identification of the myocardial specific isoenzyme of creatine phosphokinase (MB form) in the diagnosis of acute myocardial infarction. Circulation 47, 263-269 (1973)

26) Weidler, D.J.: Myocardial damage and cardiac arrhythmias after intracranial hemorrhage. A critical review. Stroke 5, 759-764 (1974)

27) Estanol, B.V., Loyo, M.V., Mateos, J.H., Foyo, E., Corneijo, A. and Guevara, J.: Cardiac arrhythmias in experimental subarachnoid hemorrhage. Stroke 8, 440-447 (1977)

28) Yamori, Y., Ohtaka, M., Horie, R., Nara, Y. and
Ooshima, A.: Significance of spontaneous hypertension for cardiac hypertrophy and myocardial lesions in stroke-prone SHR. Japan. Heart J. 20, Supp. I, 267-269 (1979)

29) Ceremuzynski, L., Stazemska-Barzak, K. and Herbaczynska-Cedro, K.: Cardiac rhythm disturbances and the release of catecholamines after acute coronary occlusion in dogs. Cardiovasc. Res. 3, 190-197 (1969)

30) Sommers, H.M. and Jennings, R.B.: Ventricular fibrillation and myocardial necrosis after transient ischemia: Effects of treatment with oxygen, procainamide, reserpine and propranolol. Arch. Intern. Med. 129, 780-789 (1972)

31) Wit, A.L., Hoffman, B.F. and Rosen, M.R.: Electrophysiology and pharmacology of cardiac arrhythmias IX: Cardiac electrophysiologic effects of beta-adrenergic receptor stimulation and blockade. Part A. Am. Heart J. 90, 521-533 (1975)

32) Gercken, G. and Döring, R.: The mechanism of the anoxic heart after surgical denervation or reserpine pretreatment. J. Mol. Cell Cardiol. 5, $275-286$ (1973)

33) Reimer, K.A., Rassmussen, M.M. and Jennings, R.B.: Reduction by propranolol of myocardial necrosis following temporary coronary occlusion in dogs. Circ. Res. 33, 353-365 (1973)

34) Sakai, K. and Spieckermann, P.G.: Effects of reserpine and propranolol on anoxia-induced enzyme release from isolated perfused guineapig heart. Naunvn Schmiedebergs Arch. Pharmacol. 291, 123-130 (1975)

35) Fox, K., Welman, E, and Selwyn, A.: Myocardial infarction in dogs: Effects of intravenous propranolol. Am. J. Cardiol. 45, 769-774 (1980)

36) Hunt, D. and Gore, 1.: Myocardial lesions following experimental intracranial hemorrhage: Prevention with propranolol. Am. Heart J. 83, 232-236 (1972)

37) Cruickshank, J.M., Neil-Dwyer, G. and Lane, J.: The effect of oral propranolol upon the ECG changes occurring in subarachnoid hemorrhage. Cardiovasc. Res. 9, 236-245 (1975)

38) Offerhaus, L. and Van Gool, L.: Electrocardiographic changes and tissue catecholamines in experimental subarachnoid haemorrhage. Cardiovasc. Res. 3, 433-440 (1969)

39) Hawkins, W.E. and Clower, B.R.: Myocardial damage after head trauma and simulated intracranial hemorrhage in mice: the role of the autonomic nervous system. Cardiovasc. Res. 5 , 524-529 (1971) 